\title{
THE CHRONIC LEUKAEMIAS
}

Peter Jacobs, Lucille Wood

\section{INTRODUCTION}

Medical students, little more than a quarter of a century ago, observed first hand the inevitable progression and early demise that characterised acute leukaemia, whether this occurred in children or adults. In contrast, there were occasional patients with a chronic variant that advanced more slowly and was compatible with extended survival albeit despite a steadily

deteriorating quality of life. Since that time progress in management has been both impressive and far reaching, first with the introduction of effective chemotherapeutic agents that made disease control possible and more recently with evidence that cure is now a realistic goal for treatment, at least in a substantial number of patients. Consequently physicians, and particularly haematologists, must ensure that the available rsources are correctly allocated so that the maximum number of individuals, whether they be adults or children, benefit from the new knowledge accrued during the last decade.

The ever widening acceptance of the need to review older practices raises ethical questions. Should limited resources be expended on people with malignant disease? Is the treatment worse than the cure? Are the results of more aggressive therapy programmes sufficiently good to justify their continuation? Do

multidisciplinary and specialised centres infringe in any way upon the role of the private or general physician? Is it possible to justify a watch-and-wait approach, even in selected groups?

Conversely, and seldom debated with the same enthusiasm, is the issue of exposing a patient with chronic leukaemia to single agent therapy which is, at best, palliative and could be leukaemogenic. Such a choice may be correct but that decision requires a degree of experience seldom found when only small numbers of patients are treated. Similarly, and of even greater concern, is the not infrequent practice of failing to provide reliable information about the alternative treatment options, at least one of which is potentially curative.

To place in perspective some of these relevant issues the clinical presentation for each disease will be outlined and followed by a brief summary of diagnostic methods, emphasising areas where new information is available. Conventional management will then be contrasted with currently preferred therapy emphasising the need for recognising and stratifying patients to appropriate options according to prognostic factors. Where it is relevant prevailing controversy will be commented on in an attempt to provide a perspective for primary care physicians and paramedical professionals in advising their patients about treatment.

\section{Summary \\ The slow progression of both chronic granulocytic and lymphocytic \\ leukaemia, when compared to their acule counterparts, has been used as an argument to support less aggressive therapy or even, in some instances, a watch-and-wait policy. This conservative approach is bolstered by a number of observations including the ease with which haematologic control can initially be achieved, the older age of patients with the lymphocytic variant and the paucity of controlled data showing that long disease-free survival or cure can result from the use of aggressive treatment. Given these circumstances, it is not surprising that many such individuals are managed outside specialised centres using a variety of agents and schedules, both of which may, on occasions, be inappropriate. Accumulating evidence suggests a need to reconsider these practices since cure is now possible in selected patients with chronic granulocytic leukaemia while the use of multi-drug regimens in the lymphatic form can significantly improve survival. These advances are the result of carefully conducted clinical trials involving many individuals the world over and constitute the basis for advocating early referral to those institutions where all the necessary expertise is available.}

Finally, the argument will be advanced that, with improved understanding in the biology and treatment of these diseases, it is advisable for patients to be referred to specialist haematologists in preference to the older practice of single agent administration by general practitioners or physicians.

\section{CHRONIC GRANULOCYTIC \\ LEUKAEMIA}

The clinical presentation is variable since the disease may be found in child hood but has an incidence between $30-40$ years in Blacks, $40-60$ years in those of mixed ancestry and 50-70 years in Whites (1). In the post-pubertal patient the first abnormality detected may be on a routine blood count where an absolute and left shifted granulocytosis is uncovered. More usually a mild degree of anaemia may give rise to symptoms while a dragging sensation in the back or left upper quadrant discomfort may draw attention to a spleen which is frequently moderately and occasionally massively enlarged (1). The remainder of the physical examination is usually negative although in advanced disease cachexia occurs. We have been unable to confirm reports about prominent lymphadenopathy in black patients (2).

The clinical course typically has three stages. The first, which is designated the stable phase, has a median duration of about 36 months and symptoms are easy to control with therapy. In the second, defined as acceleration, the blood count may not change markedly but there is deterioration in performance status, weight loss, sweating, increasing organ enlargement and the need for larger quantities of chemotherapy. The third phase is blastic transformation and here it is essential to recognise that the acute leukaemia may be lymphoblastic in $25 \%$ of cases with important implications for management.

Diagnosis rests primarily on finding a raised white count in which the differential distribution usually has two peaks with one in mature neutrophils and a second in the myelocytes: occasional blast cells will frequently be circulating. In the bone marrow there is massive granulocytic hyperplasia with loss of fat spaces, increase in reticulin and megakaryocytic hyperplasia with striking morphologic abnormalities.

Chromosomal studies will typically show the balanced reciprocal translocation of material between the long arms of chromosome 9 and 22 (3) and no patient should have this diagnosis accepted without supportive cytogenetic data. The current evidence would suggest that the Philadelphia-negative variant is more properly considered among the myelodysplastic syndromes and then specifically classified as chronic myelomonocytic leukaemia.

Biochemical profile is not particularly helpful other than demonstrating a raised urate level and thereby emphasising the importance of administering the xanthineoxidase inhibitor, allopurinol, before commencing chemotherapy.

Where cytogenetic studies are not readily available the low levels or absence of leukocyte alkaline phosphatase in granulocytes is helpful. Of interest, but not essential to the haematologic diagnosis, are studies correlating changes in the distribution of plasminogen activator secreted by the leukaemic blasts in culture (4) where preliminary studies suggest that prominence of the tissue species of enzyme over its urokinase form predicts for disease acceleration and blastic transformation.

Conventional management was, for a long time, oral myleran. Starting dose with this agent vary with the white cell count but are usually in the range of 4-6 $\mathrm{mg}$ and are then titrated to keep the total granulocyte count around $10 \times 10^{9} / \mathrm{L}$ at which time the patient should have an 
impalpable spleen. The dangers of myleran have long been recognised and recent studies have re-emphasised these (5): accordingly hydroxyurea is currently the preferred agent. With the latter drug it should be appreciated that a marked increase in mean cell volume is a characteristic finding and does not constitute an indication for measurement or administration of folate or vitamin B 12 .

It is during this chronic stable phase that management is easy with either drug administration or radiotherapy (6) so that both general practitioners and physicians have felt justified in holding on to their patients. This is a critical phase in determining survival and it is therefore unfortunate that these individuals are not clearly informed that they are receiving palliative management. Conversely other options, that include bone marrow transplantation in suitably selected patients and which offer a chance for cure although associated with greater degrees of morbidity and mortality, are often never even discussed.

During the accelerated or blastic phase he outlook is poor and there is an understandable degree of nihilism even in centres that have developed expertise in this area. A particular problem is rapid splenic enlargement frequently associated with exquisite pain as a result of multiple infarctions. Prior splenectomy is the only way to avoid this complication but the routine use of this operation remains controversial. Management for patients during this short terminal period, which seldom extends beyond 3 months, is limited to analgesia although on occasions symptomatic relief can be achieved with the oral cytotoxic drug combination of etoposide and an anthracyline antibiotic.

The preferred management is immediate referral to a centre having an established protocol for the investigation and accurate characterisation of the disease. The initial treatment is administration of hydroxyurea to return the expanded granulocyte mass towards normal. Based on a retrospective udy (Jacobs, Wood and Dent unpublished) splenectomy is reserved for selected patients rather than, as previously employed, on a routine basis.

In those patients under 45 years with a suitable donor allogeneic bone marrow transplantation is the recommended form of treatment (7). There are subtleties to this procedure that include differences in the preparative or conditioning regimens that may be ablative radiotherapy, chemotherapy or combinations of the two Furthermore ex vivo manipulation of the graft may be undertaken since T-cell depletion will reduce the incidence and severity of acute and chronic graft-versushost disease (GvHD) but there are suggestions that this manipulation may be associated with a higher leukaemic relapse rate. Alternatively whole marrow may be infused and post-transplantation immunosuppressive regimens employed to modulate the expression of GvHD, particularly since this phenomenon may have some anti-leukaemic benefits: a currently favoured regimen is the combination of ciclosporin and methotrexate.

Those patients who are not eligible for bone marrow transplantation, who cannot be adequately informed of the risks involved or who elect not to be grafted can be managed with biological immune response modulation using recombinant alpha interferon (8), with or without additional hydroxyurea. Earlier suggestions that autologous bone marrow transplantation may delay the onset of accelerated or blastic phase have not been confirmed. However studies are presently in progress to determine whether it is possible to eradicate the leukaemic clone by long-term bone marrow culture using the Dexter technique (9) and then to reinfuse adequate numbers of these "purged" haematopoietic stem cells after aggressive therapy to eradicate the leukaemic process in the patient.

During the accelerated or blastic phase allogeneic bone marrow transplantation has been shown to have a substantial response rate and a number of such patients, albeit considerably less than those transplanted during the stable phase, will achieve long disease-free survival and certainly some will be cured (10).

The ethical considerations are particularly important since cure is now a realistic goal for therapy. Many of these patients are young and there can be no justification for not offering them the opportunity to consider marrow grafting should a suitable donor be available. The ability to understand the procedure is important since this more aggressive approach has a higher morbidity and mortality in the short term than single drug palliative therapy. However since the long term results are clearly superior there can be no question that transplantation programmes should be major commitments in university centres. Another aspect of importance is the misconception that referral of patients in this way conflicts with what is best for that individual. Exactly the opposite is true. Thus optimum treatment by a multidisciplinary group protects the patient from inappropriate treatment, balances available options carefully against prognostic factors including social circumstances and then provides the patient with a comprehensive explanation of the choices. Only under these circumstances can properly informed consent be obtained and this is a necessary step for the voluntary participation in protocol studies approved by Ethics and Research Committees.

\footnotetext{
Summary

In the suitably selected young patient with a donor allogeneic bone marrow transplantation offers a chance for cure and no individual should be denied the opportunity to enter these programmes. Without a donor hydroxyurea with or without recombinant alpha interferon is preferable to busulphan. Routine splenectomy is no longer recommended but the operation retains a role on the
}

basis of clearly defined indications. In the accelerated or blastic phase allogeneic bone marrow transplantation can be effective on occasions but the majority of these patients are best managed palliatively: here an experienced multidisciplinary team can play an important role in providing good quality terminal care.

\section{CHRONIC LYMPHOCYTIC LEUKAEMIA}

The clinical presentation is variable but characteristically has a median peak at approximately 60 years of age, with no significant sex or race difference.

Presentation may be fortuitous where an elevated absolute lymphocyte count is found at routine examination or minor degrees of organ enlargement revealed during an executive medical examination. At the other end of the spectrum there may occur recurrent infections which are usually respiratory The first visit may be as a result of massive enlargement of superficial as well as deep lymph nodes or moderate degrees of hepato-splenomegaly.

Occasionally atypical variants may be encountered that include

prolymphocytic and hairy cell

leukaemia where the spleen may extend right into the true pelvis.

Diagnosis generally requires an elevated absolute lymphocyte count although with immunopherntyping (11) a much greater degree of conidence is possible at counts that may remain in the normal range having only a relative lymphocytosis present. These studies also provide a much more secure basis for the clonal nature of these disorders. It needs to be appreciated that with current sub-division of patients as a basis for therapy, exemplified by the use of interferon in those with hairy cell leukaemia, accuracy at this initial stage is of increasing importance. Classification may be refined with cellular and molecular biologic techniques that include the demonstration of rearranged immunoglobulin and $\mathrm{T}$-cell receptor genes. Immunoglobulin levels in the plasma are generally reduced and may be associated with monoclonal spikes or secretion of light-chains in the urine.

Bone marrow aspiration shows an increased number of lymphocytes whereas trephine biopsy provides valuable information on staging (12). Cytogenetic studies have been less well developed in the chronic lymphoproliferative disorders than in granulocytic tumours but nevertheless all such patients should be examined since the presence of karyotypic defects predict for more aggressive clinical course.

On the basis of laboratory data patients are staged $(13,14)$ and it is particularly important to define performance status (15).

Conventional management is based on the observation that early stage disease, particularly in the elderly, is slowly progressive and often relatively stable. Under these circumstances a watch-andwait attitude may be appropriate. More 
usually however patients require therapy because of an accelerating clinical tempo or the presence of extensive enlargement of lymph nodes or spleen and have traditionally received the alkalating agent chlorambucil (16) with or without prednisone: those who failed to respond needing additional drugs such as cyclophosphamide or occasionally cytosine arabinoside (17). Low-dose total body irradiation has been demonstrated in prospective randomised study to be without significant side effects and approximately equivalent to this kind of low-level palliative chemotherapy (18).

The preferred management is best determined by early referral to a centre that has accumulated a substantial experience over many years and where all the necessary diagnostic techniques are available. Only under these circumstances can patients be properly staged and benefit from rationally based therapy, whether this be expectant or definitive. The older practice of referral only once a refractory clone has emerged, haematopoietic reserve is eroded from prior inappropriate palliative therapy and the patients are generally in poor clinical condition is to be deprecated.

In the newly diagnosed patient an essential determinant for treatment is the extent or stage of the disease. The various procedures on which this depends should be carried out by a multidisciplinary group and take into account any associated medical conditions as reflected in the performance status as well as the socioeconomic circumstances. Among the other variables that receive particular attention are the rate at which the disease is progressing and the patients age. Only once all of these have been considered is it possible to offer an appropriate form of comprehensive management.

In those patients with minimal disease and in whom careful surveillance has documented clinical and haematologic stability there is a reasonable argument for observation alone: the watch-and-wait policy.

Where treatment is justified prospective controlled data (19) have demonstrated the benefits of a four drug regimen containing cyclophosphamide, adriamycin, vincristine and prednisone that goes by the acronym of $\mathrm{CHOP}$. Preliminary studies using high dose cyclophosphamide (Jacobs unpublished) have demonstrated efficacy with prompt return of the bone marrow to normal but it is yet to be determined whether such patients will requmaintenance or even that survival is significantly prolonged. These more aggressive approaches are consistent with a similar shift in managing indolent or low grade malignant lymphoma and multi-drug regimens evaluated at the National Cancer Institute document a significant increase in complete remission rates and disease-free survival (20).

At least three other options are available.

Firstly there is precedent for allogeneic bone marrow transplantation in a small group of selected individuals whereas autologous grafting with appropriate ex vivo purging with monoclonal antibodies is an encouraging alternative (21).

Secondly biological immune response modulation is being actively developed. In this regard monoclonal antibodies may be administered in vivo and such serotherapy is theoretically sound but remains an experimental approach. Conversely recombinant alpha interferon is clearly established in some of the variants most notably hairy çell leukaemia (22). It has recently been shown that this agent is also effective in the prolymphocytic sub-type (23) but it remains to be established to what extent these agents will become generally applicable to chronic lymphocytic leukaemia and at the present time there is no uniformity about the choice of agent or the schedule likely to be most effective.

Thirdly, either as initial therapy or once drug treatment has failed, sequential halfbody irradiation is clearly of benefit (24). In this context it appears that high doses may be given with preservation of haematopoietic reserve provided that exposure rates are low. It is clear that, here again, considerable experience is necessary to optimally use this more recently introduced form of management.

Another area that is destined to play an important role in managing these patients is the availability of recombinant growth factors. Thus the granulocyte: macrophage-colony stimulating factor or interleukin 3, which is also known as multipoietin, offer potent means for stimulating marrow regeneration following the use of myelosuppressive drugs or radiotherapy. Clearly these biological products will make it possible to escalate dosage and simultaneously reduce the hazards of neutropenia and thrombocytopenia.

Splenic enlargement also requires comment. Thus where there is associated immune destruction of blood cells that have responded inadequately to corticosteroids, splenectomy is an effective means of re-establishing control over the disease. However these patients are significantly immunocompromised and judgement as to the timing of this procedure requires a great deal of experience. Furthermore in certain of the variants, most notably hairy cell leukaemia, splenectomy is first step in managing the patient (25) although it is possible that interferon may circumvent this surgical procedure in some individuals. The results of ongoing clinical trials to define the respective role of these two approaches to splenomegaly will influence future practice.

The ethical considerations are

noteworthy. These improving results place a direct responsibility on referral centres to ensure that investigation and staging are comprehensive and then to encourage entry into protocols designed to advance knowledge concurrently with selection of optimum treatment for each person. This renewed interest in lymphocytic leukaemia derives from the clear demonstration that survival can be improved by multi-drug regimens. At the same time proper stratification will identify and divert from aggressive therapy that sub-population where observation or only palliative medication is more appropriate.

The role of the general practitioner or physician should not be overlooked since many of these patients are elderly and have associated ailments that can perfectly well be managed in the home circumstances. In this latter regard there needs to be a clear understanding as to the special hazards of the disease, potential complications of superimposed chemotherapy and the particular dangers of infections that may well be trivial in the general population. The latter is sxemplified by bacterial pneumonia which is often rapidly fatal in the immunocompromised patient. Approached in such a balanced way the patients best interests are most likely to be served.

\section{Summary}

In the elderly patient with an indolent or slowly progressive form of this disease the watch-and-wait approach may well be acceptable. However where treatment is necessary it is essential to use all diagnostic methods to stratify patients into groups who justify only palliation in contrast to others where aggressive management is clearly indicated. In the former group either total body irradiation or a combination of chlorambucil and prednisone are roughly equivalent: the first having advantages because patients need not take drugs and are spared the risk of corticosteroid administration. In the younger individual, particularly with high bulk or advancing disease, multidrug combination chemotherapy is emerging as a means for significantly improving survival. In an extension of this approach escalation in dosage, often of a single agent, with autologous bone marrow transplantation to circumvent myelotoxicity or alternatively allografting are gathering impetus as procedures that should be studied in academic centres to define, in carefully selected sub-groups, their curative potential.

\section{CONCLUSIONS}

In patients with the chronic leukaemias the time has come to critically review the conventional wisdom of automatically palliating everybody who needs treatment with only a single drug (26). It is true that initially most will do well but there is typically concurrent disease advancement, loss of haematopoietic reserve and decrease in performance status. Unfortunately it is only at this late stage that most individuals are referred to academic centres so it is not surprising that results are then poor.

Conversely recent advances have established that cure is a realistic goal in certain young patients with chronic granulocytic leukaemia seen during the chronic stable phase. Similarly drug 
combinations for the chronic lymphoproliferative disorders have a significantly superior disease-free survival in comparison to more conservative approaches.

In order that each individual can be optimally evaluated, using all modern diagnostic techniques and then rational decisions made about treatment taking into account currently recognised prognostic factors, it would seem most responsible to arrange referral to a multidisciplinary group that have accumulated the necessary experience over prolonged periods of time. This approach has the additional advantage that patients will be entered on properly structured trials and it is well recognised that, for any given form of treatment, they will do significantly better when entered into a carefully supervised protocol than community treated individuals.

It is apparently also necessary to restate the fact that such programmes make allowance for all the variables that include age and social circumstance which, particularly in local practice, may become xclusions from randomised study. rurthermore this approach does not exclude participation of the general practitioner or physician since both will continue to see their patients for minor ailments. It is also clearly necessary to appreciate the geographical constraints that exist in this country. Thus, while the recommendation stands for referral to specialised clinics, local custom or the distances involved may complicate the delivery of optimal therapy and here there exists a place for compassionate care and the use of palliative programmes.

Undoubtedly the contemporary management of chronic leukaemia has become a much more exacting requirement than in the past and, all other arguments notwithstanding, the current evidence provides a compelling reason for acknowledging that the investigation and treatment of these patients now falls clearly within the ambit of haematologic ecialists. To ensure that all aspects of their care receive proper consideration a multidisciplinary approach is to be favoured. This includes a sound knowledge of cancer chemotherapy, participation by social workers and radiotherapists in a clinic that has ready access to the sophisticated practices of cell support and bone marrow transplantation while additional responsibilities are the study and use of recombinant growth factors and biologic immune response modifiers. The future is encouraging and these directions more than justify a reasonable allocation of clinical and research resources to university hospitals that have made a commitment to the systematic study and treatment of haematologic malignancies.

\section{Acknowledgements}

We thank our colleague, Nicholas Novitzky, for his helpful comments in preparation of the manuscript, Keren
Hounsell and Nerima Ismail for bibliograph assistance and Dorothy Banner for preparation and typing of the manuscript.

\section{REFERENCES}

I. Jacobs P., King H.S., Dent D. M. Chronic granulocytic leukaemia. A 10-year experience in the Black, Coloured and White populations of the south-western Cape Province. S Afr Med J 1983; 63: 879-882.

2. Leibowitz M,R, Derman D.P., Jacobson R., Stevens K., Katz J. Chronic myeloid leukaemia in South African Blacks. $S$ Afr Med J 1975; 50: 2035-2037.

3. Sokal J.E., Gomez G.A. The philadelphia chromosome and philadelphia chromosome mosaicism in chronic granulocytic leukaemia. J Clin Oncol 1986; 4: 104-111.

4. Wilson E.L., Jacobs P., Oliver L. Plasminogen activator as a prognostic factor in haematological malignancies. In: Neth, Gallo, Graves, Janka, (eds) Modern Trends in Human Leukaemia VI. Haematology and Blood Transfusion 1985; 29: 197-199.

5. Key N.S., Kelly P.M.A., Emerson P.M., Chapman R.W.G., Allan N.C., McGee JO'D. Oesophageal varices associated with busulphan-thioguanine combination therapy for chronic myeloid leukaemia. Lancet 1987; ii: $1050-1052$

6. Jacobs P., Dubovsky D., King H.S., Sealy R. Splenic irradiation in the management of chronic granulocytic leukaemia. Central Afr $J$ Med 1975; 21: 207-210

7. Marmont A.M. Allogeneic bone marrow transplantation for chronic granulocytic leukemia: progress and controversies. Acta Haemat 1987; suppl 1: 181-186

8. Kurzrock R., Talpaz. M., Kantarjian H., Walters R., Saks S., Trujullo J.M. Gutterman J.U. Therapy of chronic myelogenous leukaemia with recombinant interferon-y. Blood 1987; 4: 943-947.

9. Coulombel L., Kalousek D.K., Eaves C.J., Gupta C.M., Eaves A.C. L.ong-term marrow culture reveals chromosomally normal hematopoietic progenitor cells in patients with philadelphia chromosome-positive chronic myelogenous leukemia. $N$ Engl J Med 1983; 308: 1493-1498

10. McGlave P.B., Arthur D.C., Weisdorf D., Kim T., Goldman A., Hurd D.D., Ramsay N.K.C., Kersey J.H. Allogeneic bone marrow transplantation as treatment for accelerating chronic myelogenous leukemia. Blood 1984; 63: 219-222

11. Jacobs P. Immunophenotypic classification of lymphoblastic leukaemia and lymphocytic lymphoma an experience in the southwestern area of the Cape Province of South Africa. In: Greaves M.F., Chan L.C. eds Epidemiology of Leukaemia and Limphoma 1984: 93-101

12. Bartl R., Frisch B., Burkhardt R. Hoffmann-Fezer G., Demmler K., Sund M. Assessment of marrow trephine in relation to staging in chronic lymphocytic leukaemia. $\mathrm{Br}$ $J$ Haematol 1982; 51: 1-15.

13. Rai K.R., Sawitsky A., Cronkite E.P., Chanana A.D., Levy R.B., Pasternack B.S. Clinical staging of chronic lymphocytic leukemia. Blood 1975; 46: 219-234.

14. Binet J.L., Catovsky D., Chandra P., Dighiero G., Montserrat E., Rai K.R., Sawitsky A. Chronic lymphocytic leukaemia proposals for a revised prognostic staging system. Br J Haematol 1981; 48: 365-367.
15. Mor V., Laliberte L., Morris J.N., Wiemann $M$. The Karnofsky performance status scale. An examination of its reliability and validity in a research setting. Cancer 1984; 53. 2002-2007.

16. Sawitsky A., Rai K.R., Glidewell O., Silver R.T., and participating members of CALGB (Cancer and Leukaemia Group B). Comparison of daily versus intermittent chlorambucil and prednisone therapy in the treatment of patients with chronic lymphocytic leukaemia. Blood 1977; 50: 1049-1059.

17. Keller J.E., Knospe W.H., Raney M., Huguley C.M., Johnson I.., Bartolucci A.A., Omura G.A. Treatment of chronic lymphocytic leukaemia using chlorambucil and prednisone with or without cycle-active consolidation chemotherapy. A South Eastern Cancer Study Group trial. Cancer 1986; 58: 1185-1192.

18. Jacobs P., King H.S. A randomised prospective comparison of chemotherapy to total body irradiation as initial treatment for the indolent lymphoproliferative diseases. Blood 1987; 69: 1642-1646.

19. French Co-operative Group on Chronic Lymphocytic Leukaemia. Effectiveness of "CHOP" regimen in advanced untreated chronic lymphocytic leukaemia. Lancet 1986 ; i: $1346-1349$

20. Young R.C., Longo D.L., Glatstein E., Ihde D.C., Jaffe E.S., DeVita Jr V.T. The treatment of indolent lymphomas: watchful waiting versus aggressive combined modality treatment. Sem in Haemalol 1988; In press.

21. Macintyre E.A. The use of monoclonal antibodies for purging autologous bone marrow in the lymphoid malignancies. Clin in Haematol 1986; 15: 249-267

22. Golomb H.M., Jacobs A., Fefer A., Ozer H., Thompson J., Portlock C., Ratain M. Golde D., Vardiman J., Burke J.S., Brady J., Bonnem E., Spiegel R. Alpha-2 interferon therapy of hairy cell leukaemia: a multicentre study of 64 patients. $J$ Clin Oncol 1986; 4: 900-905.

23. Jacobs P., Le Roux I., Wood L., Bolding E. Interferon response in B-cell prolymphocytic leukaemia. Br J Haematol 1987; 65: 375-376.

24. Tobias J.S., Richards J.D.M., Blackman G. M., Joannides T., Trask C.W.L., Nathan J.I. Hemibody irradiation in multiple myeloma. Radiother Oncol 1985; 3: 11-16.

25. Jacobs P., King H.S., Dent D.M., Westhuizen N. vd. Splenectomy as primary treatment for hairy cell leukaemia. Br J Surg 1987: 74: 1169-1170.

26. Jacobs $P$. Management of the chronic leukaemias. $S A f r$ Med $J 1988$; In press.

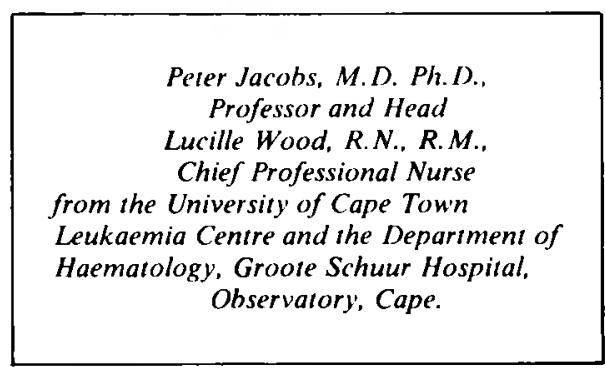

Supported by the University of Cape Town Leukaemia Centre and Staff Research Fund. the National Cancer Association, the Medical Research Council, the Gwendoline Moore Trust, the Michael Chanani and Kaliski Bequests. 